\title{
Title
}

Neurogranin as a potential synaptic marker in the cerebrospinal fluid of patients with a first episode psychosis

\section{Authors and affiliations}

Santillo $\mathrm{AF}^{1,2^{*}}$, Lundgren $\mathrm{S}^{2 *}, \mathrm{Xu} \mathrm{C}^{3}$, Orhan $\mathrm{F}^{3}, \mathrm{H}$ Fatouros-Bergman ${ }^{1}$, Blennow $\mathrm{K}^{4,5}$, Zetterberg $\mathrm{H}^{4-7}$, Portelius $\mathrm{E}^{4,5}$, Karolinska Schizophrenia Project (KaSP)

Consortium $^{8}$, Cervenka S ${ }^{1}$, Erik G. Jönsson ${ }^{1,9}$, Erhardt $S^{3}$, G Engberg ${ }^{3}$

${ }^{1}$ Centre for Psychiatry Research, Department of Clinical Neuroscience, Karolinska Institutet, \& Stockholm Health Care Services, Stockholm County Council, Stockholm, Sweden; ${ }^{2}$ Clinical Memory Research Unit and Psychiatry, Department of Clinical Sciences, Malmö, Lund University, Sweden; ${ }^{3}$ Department of Physiology and Pharmacology, Karolinska Institutet, Stockholm, Sweden; ${ }^{4}$ Clinical Neurochemistry Laboratory, Institute of Neuroscience and Physiology, Sahlgrenska Academy at the University of Gothenburg, Mölndal, Sweden Neurochemistry, Institute of Neuroscience and Physiology, the Sahlgrenska Academy at the University of Gothenburg, Mölndal, Sweden; ${ }^{6}$ Department of Neurodegenerative Disease, UCL Institute of Neurology, Queen Square, London, UK; ${ }^{7}$ UK Dementia Research Institute at UCL, London, UK. ${ }^{8}$ Members of Karolinska Schizophrenia Project (KaSP) are listed at the end of the manuscript as collaborators. ${ }^{9}$ NORMENT, KG Jebsen Centre for Psychosis Research, Institute of Clinical Medicine. Psychiatry section, University of Oslo, Norway 
*These authors have contributed in an equal part to this publication/shared first authorship

\section{Corresponding author}

Alexander Frizell Santillo

Clinical Memory Research Unit,

Department of Clinical Sciences, Lund University

Simrisbanvägen 14 B

20502 Malmö

Sweden

alexander.santillo@med.lu.se

Telephone: +46768812731

Fax: +464033 5657

\section{Letter to the editor}

The neurodevelopmental hypothesis (Weinberger, 1987) has a prominent position in the current thinking about the pathophysiology of schizophrenia, but despite support from post-mortem neuropathology, genetics and animal models, direct in vivo human evidence of is lacking. Neurogranin is a postsynaptic protein important for calmodulin-dependent calcium signal transduction and long-term potentiation (LTP) establishment (Diez-Guerra, 2010). Measurement of this protein in cerebrospinal fluid (CSF) has repeatedly shown increased levels in Alzheimer's disease in a way that is thought to reflect ongoing synaptic degeneration. This, since CSF neurogranin correlates with other biomarkers of synaptic damage and loss of activity, such as CSF tau, hippocampal atrophy and cortical hypometabolism (Portelius et al. 2015). We examined CSF neurogranin by Meso Scale Discovery immunoassay (Portelius et al., 
2015) in 40 first episode psychosis (FEP) patients (mean age $29 \pm 7$ years, DSM-IV diagnoses) and 20 healthy controls (mean age $25 \pm 4$ years) from the Karolinska Schizophrenia Project (KaSP), an ongoing longitudinal study of FEP patients in Stockholm, Sweden (Orhan et al., 2018). The study was approved by the Regional Ethics Committee in Stockholm and conformed to the tenets of the Declaration of Helsinki. All subjects had given their written consent after receiving written and oral information. Twenty-two patients were antipsychotic-naive, and the remaining patients had been treated for a median of 16 days (interquartile range 10). Subjects underwent symptom rating using the Positive and Negative Symptoms Scale symptom rating scales (PANSS) and cognitive testing using the Measurement and Treatment Research to Improve Cognition in Schizophrenia Consensus Cognitive Battery (MCCB) (Nuechterlein et al., 2008) (for detailed clinical and demographical information see Supplementary Table 1). CSF neurogranin was lower in FEP patients (342 pg/mL \pm 207 ) compared to controls ( $427 \mathrm{pg} / \mathrm{mL} \pm 189$, Cohen's d=0.43), but this difference was not statistically significant (two-tailed independent t-test $\mathrm{p}=0.127$ ) (Figure 1). Out of the 40 FEP patients 27 were diagnosed with a schizophrenia spectrum disorder either at baseline or at follow up. As a group these had slightly lower mean CSF neurogranin concentrations $(328 \mathrm{pg} / \mathrm{mL} \pm 191)$ than the entire FEP group, still not significantly different from the mean in the healthy controls $(\mathrm{p}=0.084)$. Using multiple linear regression, with covariates chosen on criterion of descending effect sizes (see Supplementary Table 2 and 3), we found no significant effect of FEP/healthy control status on CSF neurogranin $(B=0.042, \mathrm{p}=0.766)$. In the FEP group, there were significant effects of antipsychotic treatment, associated with lower levels of CSF neurogranin $(\beta=-0.323, p=0.040)$, and smoking, associated with higher levels $(B=0.317, \mathrm{p}=0.040)$. Post hoc t-test (two-tailed, independent) of CSF 
neurogranin between medicated patients only $(\mathrm{n}=18$, mean $274 \mathrm{pg} / \mathrm{mL}, \pm=181)$ and controls showed a statistically significant difference $(p=0.015$, Cohen's $d=0.83)$. No relationships emerged between CSF neurogranin levels and symptom scores or cognitive measures. Our study, the first in its kind, does not support altered CSF neurogranin levels as a marker of synaptic degeneration which is thought to occur at the FEP stage. Notably, neurogranin is among those synaptic proteins that have been shown to be reduced in neuropathological samples in schizophrenia (Broadbelt et al., 2006, Osimo et al., 2018). As for other studies evaluating proteins in CSF as markers of altered synaptic dynamics in a developmental context, we believe that a limitation of our study is the lack of regional distinction, given that CSF-neurogranin likely reflects contribution from the entire brain (Represa et al., 1990), while both neuronal maturation processes (Huttenlocher and Dabholkar, 1997) and the pathology of FEP and schizophrenia may show anatomical restriction (Bora et al., 2011). Further, if schizophrenia origins in the development of the central nervous system, it follows that CSF evaluation of neurogranin should preferably be performed during the prodromal phase, before the onset of FEP. Thus, estimation of neurogranin during the FEP stage, as is the case of the present study, may not be optimal to evaluate i.e. disturbed synapse dynamics in this condition. Antipsychotic medicated and unmedicated FEP subjects did not differ significantly in clinical parameters (Supplementary Table 4) and although there are inherent limitations in a cross sectional comparison, our results suggests that CSF neurogranin may be altered as a consequence of even minimal exposure to antipsychotic treatment. This is well in accordance with the known effect of D2 receptor antagonism on calcium dependent signal transduction (Beaulieu and Gainetdinov, 2011), and may indicate that CSF neurogranin is a possible second messenger in dopamine D2 receptor-mediated signaling that also is accessible for in 
vivo measurements in humans. Longitudinal clinical and experimental studies are needed to examine this further. Nevertheless, this observation suggests that for CSF studies of synaptic dynamics, markers of presynaptic structures as opposed to postsynaptic are preferable, and that that antipsychotic medication appear to be an important confounder in this line of research. To summarize, the main findings of the present study is that in FEP patients, levels of the synaptic marker CSF neurogranin appear unaltered, yet there is a reduction associated with the use of antipsychotic medication.

\section{Keywords}

schizophrenia, neurogranin, neurodevelopmental hypothesis, synaptic pruning, calmodulin

\section{Collaborators}

Members of the Karolinska Schizophrenia Project (KaSP): Farde L ${ }^{1}$, Flyckt L ${ }^{1}$, Engberg $\mathrm{G}^{2}$, Erhardt $\mathrm{S}^{2}$, Fatouros-Bergman $\mathrm{H}^{1}$, Cervenka $\mathrm{S}^{1}$, Schwieler $\mathrm{L}^{2}$, Piehl $\mathrm{F}^{3}$, Agartz $\mathrm{I}^{1,4,5}$, Collste $\mathrm{K}^{1}$, Sellgren $\mathrm{CM}^{2}$, Victorsson $\mathrm{P}^{1}$, Malmqvist $\mathrm{A}^{2}$, Hedberg $\mathrm{M}^{2}$, Orhan $\mathrm{F}^{2}$

${ }^{1}$ Centre for Psychiatry Research, Department of Clinical Neuroscience, Karolinska Institutet, \& Stockholm Health Care Services, Stockholm County Council, Stockholm, Sweden; ${ }^{2}$ Department of Physiology and Pharmacology, Karolinska Institutet, Stockholm, Sweden; ${ }^{3}$ Neuroimmunology Unit, Department of Clinical Neuroscience, Karolinska Institutet, Stockholm, Sweden; ${ }^{4}$ NORMENT, KG Jebsen Centre for Psychosis Research, Division of Mental Health and Addiction, University of Oslo, Oslo, Norway; ${ }^{5}$ Department 
of Psychiatry Research, Diakonhjemmet Hospital, Oslo, Norway.

\section{Figure 1. CSF neurogranin in patients and controls.}

Values are pg/mL, bars represent mean. FEP: First Episode Psychosis, HC=Healthy Control

\section{References}

Bora, E., Fornito, A., Radua, J., Walterfang, M., Seal, M., Wood, S.J., Yucel, M., Velakoulis, D., Pantelis, C., 2011. Neuroanatomical abnormalities in schizophrenia: a multimodal voxelwise meta-analysis and meta-regression analysis. Schizophrenia research 127(1-3), 46-57.

Broadbelt, K., Ramprasaud, A., Jones, L.B., 2006. Evidence of altered neurogranin immunoreactivity in areas 9 and 32 of schizophrenic prefrontal cortex. Schizophrenia research 87(1-3), 6-14.

Diez-Guerra, F.J., 2010. Neurogranin, a link between calcium/calmodulin and protein kinase C signaling in synaptic plasticity. IUBMB Life 62(8), 597-606.

Huttenlocher, P.R., 1979. Synaptic density in human frontal cortex - developmental changes and effects of aging. Brain research 163(2), 195-205.

Nuechterlein, K.H., Green, M.F., Kern, R.S., Baade, L.E., Barch, D.M., Cohen, J.D., Essock, S., Fenton, W.S., Frese, F.J., 3rd, Gold, J.M., Goldberg, T., Heaton, R.K., Keefe, R.S., Kraemer, H., Mesholam-Gately, R., Seidman, L.J., Stover, E., Weinberger, D.R., Young, A.S., Zalcman, S., Marder, S.R., 2008. The MATRICS Consensus Cognitive Battery, part 1: test selection, reliability, and validity. The American journal of psychiatry 165(2), 203-213. 
Orhan, F., Fatouros-Bergman, H., Goiny, M., Malmqvist, A., Piehl, F., Karolinska Schizophrenia Project, C., Cervenka, S., Collste, K., Victorsson, P., Sellgren, C.M., Flyckt, L., Erhardt, S., Engberg, G., 2018. CSF GABA is reduced in first-episode psychosis and associates to symptom severity. Mol Psychiatry 23(5), 1244-1250. Osimo, E.F., Beck, K., Reis Marques, T., Howes, O.D., 2018. Synaptic loss in schizophrenia: a meta-analysis and systematic review of synaptic protein and mRNA measures. Mol Psychiatry.

Portelius, E., Zetterberg, H., Skillback, T., Tornqvist, U., Andreasson, U., Trojanowski, J.Q., Weiner, M.W., Shaw, L.M., Mattsson, N., Blennow, K., Alzheimer's Disease Neuroimaging, I., 2015. Cerebrospinal fluid neurogranin: relation to cognition and neurodegeneration in Alzheimer's disease. Brain : a journal of neurology 138(Pt 11), 3373-3385.

Represa, A., Deloulme, J.C., Sensenbrenner, M., Ben-Ari, Y., Baudier, J., 1990. Neurogranin: immunocytochemical localization of a brain-specific protein kinase $\mathrm{C}$ substrate. The Journal of neuroscience : the official journal of the Society for Neuroscience 10(12), 3782-3792.

Weinberger, D.R., 1987. Implications of normal brain development for the pathogenesis of schizophrenia. Archives of general psychiatry 44(7), 660-669. 